\title{
Endogenous sex hormones and bone mineral density among community-based postmenopausal women
}

\author{
Sean Murphy, Kay-Tee Khaw, Mary Jane Sneyd ${ }^{*}$ and Juliet E. Compston' \\ Clinical Gerontology Unit, Addenbrooke's Hospital, University of Cambridge and ${ }^{2}$ Department of \\ Medicine, University of Cambridge Clinical School, Cambridge, UK
}

\begin{abstract}
Summary: In order to describe the relationships between endogenous sex hormones and bone mineral density in healthy postmenopausal women, we carried out a cross-sectional study of 90 community-based women, all at least one year since their last menstrual period (mean $9.6 \pm 4.9$ years, range 1-22) and with a serum oestradiol level less than $100 \mathrm{pmol} / \mathrm{l}$. None was currently using hormone replacement therapy. Serum oestradiol, testosterone, sex hormone binding globulin, dehydroepiandrosterone sulphate, and androstenedione were measured using standard techniques. Free oestradiol and testosterone indices were derived as the ratio of total hormone to sex hormone binding globulin, respectively. Total body, spine and hip bone mineral density $\left(\mathrm{g} / \mathrm{cm}^{2}\right)$ were measured by dual energy X-ray absorptiometry.

Significant positive correlations were found between the free oestradiol and testosterone indices and bone mineral density at all sites. These relationships remained significant for the free oestradiol index after adjustment for age and body mass index. By stepwise multiple regression analysis, the free oestradiol index was an independent predictor of total body, spine and hip bone mineral density, accounting for $4-17 \%$ of the variance.

These findings suggest an independent positive relationship between endogenous free oestradiol and total body, spine and hip bone mass even in the late postmenopause.
\end{abstract}

\section{Introduction}

Osteoporotic fractures are a major cause of morbidity and mortality amongst postmenopausal and elderly women. Low bone mass is an important determinant of skeletal fragility and fracture risk. ${ }^{1,2}$ Several lines of evidence point to a pivotal role for sex steroids in the pathogenesis of low bone mass. Ovarian failure following menopause is associated with an accelerated rate of bone loss. ${ }^{3,4}$ Oestrogen replacement therapy prevents postmenopausal bone loss, ${ }^{5}$ increases bone mineral density in established osteoporosis, ${ }^{6}$ and is associated with a reduced fracture risk. ${ }^{7-9}$

There is considerable inter-individual variation in the rates of postmenopausal bone loss and the responsible factors are not fully understood. It is possible that differences in the levels of endogenous sex hormones may be important. Case-control

Correspondence: Sean Murphy, M.B., M.R.C.P.I., M.R.C.P.(UK), Clinical Gerontology Unit, F\&G Block, Level 2, Addenbrooke's Hospital, Cambridge CB2 2QQ, UK.

*Present address: Department of Preventive and Social Medicine, University of Otago Medical School, Dunedin, New Zealand.

Accepted: 1 June 1992 studies comparing sex hormone levels among hip and vertebral fracture cases with controls have

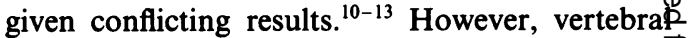
bone mineral density has been positively correlate $\vec{\Phi}$ with endogenous sex hormone levels in pre- to early postmenopausal women, ${ }^{14-16}$ and similar finding have been reported at the radius for postmeno? pausal women. ${ }^{15,17,18}$

There is a paucity of data relating endogenous sex hormones and bone mineral density in normal? community-based postmenopausal women. Onl one previous report has examined these relation? ships for hip bone mineral density, ${ }^{16}$ and never, to the best of our knowledge, for total body bone mineral density. In this paper we report the rela tionships between endogenous sex hormones anc. total body, spine and hip bone mineral density in 8 community-based sample of healthy postmenopausal women.

\section{Materials and methods}

\section{Subjects}

The subjects in this report were recruited between 1989 and 1990 into a pilot validation study compar? 
ing different dietary measurement methods. Community-dwelling women aged between 45 and 65 years, identified from two general practice registers in Cambridge, were recruited by letter. About $20 \%$ of those approached agreed to participate $(n=$ 169) and they were requested to continue their usual dietary intakes during the study period. These women were unselected as to their health status. Subsequently, 141 of these women agreed to undergo bone mineral density measurement. Of these, 90 were postmenopausal as assessed by absence of menstrual bleeding for at least one year and a serum oestradiol level less than $100 \mathrm{pmol} / \mathrm{l}$. These 90 subjects form the basis of this report. None was currently taking hormone replacement therapy, and all were ambulatory and enjoyed good general health. Subjects provided informed consent and the research protocol was approved by the hospital ethical committee. Weight and height were measured by trained observers and body mass index was calculated as weight in $\mathrm{kg} /$ (height in metres). ${ }^{2}$

\section{Hormone assays}

Non-fasting blood for the sex hormone assays was drawn between 0900 and 1500 hours and the serum removed and stored at $-70^{\circ} \mathrm{C}$ until assayed. Total oestradiol was measured by radioimmunoassay using the DPC double antibody kit (Diagnostic Products Corporation, Los Angeles, CA 90045, USA). For analysis, we used measured values though this assay is less accurate in discriminating values below $20 \mathrm{pmol} / \mathrm{l}$. For postmenopausal subjects the intra-assay coefficient of variation was up to $10 \%$. Total testosterone was measured by radioimmunoassay after extraction of serum using diethyl ether. The ${ }^{125} \mathrm{I}$-labelled testosterone derivative, the antibody and other reagents were provided by Dr M.J. Wheeler, St Thomas' Hospital, London SE1. The intra-assay coefficient of variation was $8-10 \%$. Sex hormone binding globulin (SHBG) was measured by immuno-radiometric assay using the Farmos kit (Pharmacia Ltd, Milton Keynes, UK). The intra- and inter-assay coefficients of variation were less than $5 \%$ and $3.8-8.4 \%$, respectively. A free testosterone index was calculated by dividing the total testosterone level by the sex hormone binding globulin level. ${ }^{19}$ We derived a free oestradiol index in a similar manner (total oestradiol/SHBG) to provide an estimate of the free biologically active hormone level. Androstenedione was measured by an ether extraction assay followed by standard radioimmunoassay using tritiated androstenedione as tracer. Intra- and inter-assay coefficients of variation were less than $5 \%$ and 3.6-9.0\%, respectively. Dehydroepiandrosterone sulphate (DHEAS) was measured by a direct radioimmunoassay using iodinated DHEAS as tracer. Intra- and inter-assay coefficients of variation were $3.0-4.8 \%$ and $6.8-8.8 \%$, respectively.

\section{Bone mineral density measurements}

Total and regional bone mineral density (BMD) were measured by dual energy X-ray absorptiometry (DEXA) using the Lunar DPX (Lunar Radiation Corp, Madison, WI, USA, software 3.1) ${ }^{20}$ Bone mineral density results are expressed as areal density in $\mathrm{g} / \mathrm{cm}^{2}$. Precision for total body bone mineral density ( 28 paired measurements in normal subjects) was $0.9 \%$. Spine (L2-L4) and hip precision (24 paired measurements in normal subjects) were $1.2 \%$ and $2.2-3.7 \%$, respectively; neck $2.5 \%$, greater trochanter $2.2 \%$ and Ward's triangle $3.7 \%{ }^{21}$

\section{Statistical analyses}

Statistical analyses were performed using the Statistical Package for the Social Sciences (SPSS$\mathrm{X})$ using the mainframe computer at the University of Cambridge. Pearson and partial correlation coefficients were computed and two-sided statistical tests were used throughout. Stepwise multiple linear regression analysis was used to determine the independent effects of the individual sex hormones.

\section{Results}

The baseline demographic, hormonal and bone density measurements for the 90 postmenopausal subjects are given in Table I. The sex hormone measurements were in the range expected for postmenopausal women.

Table II gives the Pearson correlation coefficients for the baseline study characteristics. Age was negatively correlated with DHEAS $(r=-0.34$, $P<0.01)$ and L2-L4 bone mineral density $(r=$ $-0.23, P<0.05$ ). Body mass index was positively correlated with total oestradiol and the free oestradiol index $(r=0.57, P<0.001$ and $r=0.62$, $P<0.001$, respectively), total testosterone and the free testosterone index $(r=0.23, P<0.05$ and $r=0.51, P<0.001$, respectively) and negatively with SHBG $(r=-0.29, P<0.01)$. Body mass index was also positively correlated with bone mineral density at all sites. All the regional bone density measurements as well as total body bone mineral density were highly intercorrelated. As expected the sex hormones were also highly correlated with each other.

Statistically significant positive correlations were seen between total oestradiol and the free oestradiol index and bone mineral density at each of the sites measured ( $r$ ranging from $0.26-0.43$ and 
Table I Distribution of variables in 90 postmenopausal women

\begin{tabular}{lccc}
\hline & Mean (s.d.) & Range \\
\hline Age (years) & $58.4(3.8)$ & $49-65$ & \\
Height (cm) & $163.8(66)$ & $147.0-177.0$ \\
Weight (kg) & $67.6(11.8)$ & $47.6-118.8$ \\
BMI (kg/m $\left.{ }^{2}\right)$ & $25.2(4.3)$ & $18.0-45.5$ & \\
Years postmenopause & $9.6(4.9)$ & $1-22$ & \\
Oestradiol (pmol/l) & $13.1(13.7)$ & $1-68$ & $n=90$ \\
Free oestradiol index & $0.46(0.43)$ & $0.1-2.1$ & $n=84$ \\
Testosterone (nmol/l) & $1.68(0.65)$ & $0.4-3.6$ & $n=90$ \\
Free testosterone index & $0.031(0.02)$ & $0.006-0.097$ & $n=84$ \\
DHEAS ( $\mu$ mol/l) & $1.54(1.07)$ & $0.22-4.8$ & $n=84$ \\
SHBG (nmol/l) & $63.2(25.8)$ & $28-153$ & $n=84$ \\
Androstenedione (nmol/1) & $6.04(2.2)$ & $1.0-12.5$ & $n=79$ \\
Total body BMD & $1.078(0.09)$ & $0.926-1.322$ & $n=88$ \\
L2-L4 BMD & $1.081(0.17)$ & $0.770-1.643$ & $n=90$ \\
Neck BMD & $0.871(0.11)$ & $0.648-1.224$ & $n=90$ \\
Trochanter BMD & $0.769(0.11)$ & $0.523-1.018$ & $n=90$ \\
Ward's triangle BMD & $0.753(0.14)$ & $0.488-1.222$ & $n=90$ \\
\hline
\end{tabular}

BMI = body mass index; DHEAS = dehydroepiandrosterone sulphate; SHBG = sex hormone binding globulin; $\mathrm{BMD}=$ bone mineral density $\left(\mathrm{g} / \mathrm{cm}^{2}\right)$. Calculated as total hormone/SHBG. ${ }^{19}$

$0.32-0.49$, respectively). The free testosterone index was significantly correlated also with bone mineral density at each site ( $r$ ranging from $0.23-$ $0.37)$. Less consistent and weaker correlations were seen for DHEAS and SHBG (inverse), and no correlation was found between androstenedione levels and bone mineral density. The correlation coefficients were no longer significant after controlling for age and body mass index by partial correlation analysis except for the free oestradiol index (total body BMD $r=0.21, P=0.058$; L2L4 BMD $r=0.25, P=0.025$; neck BMD $r=0.15$, $P=0.17$; trochanter BMD $r=0.21, P=0.055$; and ward BMD $r=0.22, P=0.045$ ).

To determine the independent effects of the sex hormones, they were entered into a multiple stepwise linear regression model along with age, years postmenopause and body mass index. Total oestradiol, total testosterone and sex hormone binding globulin were subsequently dropped because the free oestradiol and free testosterone indices (which were considered more biologically meaningful) were derived from these variables and also their removal did not alter the regression coefficients of the variables in the final models. It was also possible to drop age, free testosterone index, dehydroepiandrosterone sulphate, and androstenedione without altering the regression coefficients, leaving the free oestradiol index, years postmenopause and body mass index in the final models. The regression coefficients (Beta) and their standard errors are given in Table III. An independent, though modest effect, was seen for the free oeste diol index on total body, spine (L2-L4), femofake neck and Ward's triangle bone mineral density. trochanter bone mineral density, this effect justo failed to reach statistical significance $(P=0.054)$. The proportion of the variance explained by the free oestradiol index varied from 4 to $17 \%$. Theo relationship between the free oestradiol index and 0 bone density was partially confounded by body $\overrightarrow{\vec{\sigma}}$ mass index as its regression coefficients were 3 reduced by $10-40 \%$ by entering free oestradiol index and body mass index alone into the models. Despite these reductions, however, the free oestra-o응

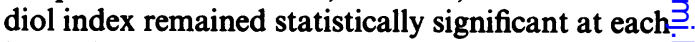
bone site except for the femoral neck $(P=0.12)$.

\section{Discussion}

Among community-based women who were on average 9.6 years postmenopause, we have shown a․ positive relationship between an index of freeN oestradiol levels and spine, hip and total body bone mineral density. While these women were volunteers who agreed to participate in dietary assess- $\omega$ ment surveys, they were unselected as regards health status, though all were ambulatory ando enjoyed good general health. Although we would $\bar{\varnothing}$ advocate some caution in generalizing our results ${ }^{?}$ to community dwelling postmenopausal women at ${ }^{\circ}$ large in view of our relatively low recruitment rate, $\frac{\mathrm{P}}{\mathbb{\Phi}}$ we believe that our findings have implications for 
Table II Pearson correlation coefficients

\begin{tabular}{|c|c|c|c|c|c|c|c|}
\hline & $B M I$ & Yrs pm & $E 2$ & FreeE2 & $T$ & FreeT & $D H E A S$ \\
\hline $\begin{array}{l}\text { Age } \\
\text { BMI } \\
\text { Yrs pm } \\
\text { E2 } \\
\text { Free E2 } \\
\text { T } \\
\text { Free T } \\
\text { DHEAS } \\
\text { SHBG } \\
\text { A } \\
\text { TBody } \\
\text { L2-L4 } \\
\text { Neck } \\
\text { Troc. } \\
\text { Ward }\end{array}$ & 0.17 & $\begin{array}{l}0.59 \\
0.07\end{array}$ & $\begin{array}{c}-0.09 \\
0.57^{\ddagger} \\
-0.03\end{array}$ & $\begin{array}{c}-0.11 \\
0.62^{\ddagger} \\
-0.04 \\
0.90^{\ddagger}\end{array}$ & $\begin{array}{c}-0.09 \\
0.23^{*} \\
-0.06 \\
0.50^{\ddagger} \\
0.35^{\dagger}\end{array}$ & $\begin{array}{c}-0.19 \\
0.51^{\ddagger} \\
-0.09 \\
0.68^{\ddagger} \\
0.82^{\ddagger} \\
0.63^{\ddagger}\end{array}$ & $\begin{array}{r}-0.34^{\dagger} \\
0.14 \\
-0.06 \\
0.42^{\ddagger} \\
0.39^{\ddagger} \\
0.40^{\ddagger} \\
0.44^{\ddagger}\end{array}$ \\
\hline & $S H B G$ & $A$ & TBody & $L 2-L 4$ & Neck & Troc & Ward \\
\hline $\begin{array}{l}\text { Age } \\
\text { BMI } \\
\text { Yrs pm } \\
\text { E2 } \\
\text { Free E2 } \\
\text { T } \\
\text { Free T } \\
\text { DHEAS } \\
\text { SHBG } \\
\text { A } \\
\text { TBody } \\
\text { L2-L4 } \\
\text { Neck } \\
\text { Troc. }\end{array}$ & $\begin{array}{c}0.16 \\
-0.29^{\dagger} \\
0.06 \\
-0.28^{\dagger} \\
-0.57^{\ddagger} \\
0.11 \\
-0.60^{\ddagger} \\
-0.20\end{array}$ & $\begin{array}{r}-0.18 \\
0.07 \\
-0.09 \\
0.41^{\ddagger} \\
0.33^{\dagger} \\
0.52^{\ddagger} \\
0.46^{\ddagger} \\
0.60^{\ddagger} \\
-0.04\end{array}$ & $\begin{array}{c}-0.15 \\
0.49^{\ddagger} \\
-0.26^{*} \\
0.43^{\ddagger} \\
0.49^{\ddagger} \\
0.15 \\
0.37^{\ddagger} \\
0.18 \\
-0.22^{*} \\
0.14\end{array}$ & $\begin{array}{r}-0.23^{*} \\
0.29^{\dagger} \\
-0.30^{\dagger} \\
0.30^{\dagger} \\
0.41^{\ddagger} \\
0.06 \\
0.33^{\dagger} \\
0.23^{*} \\
-0.28^{*} \\
0.14 \\
0.83^{\dagger}\end{array}$ & $\begin{array}{c}-0.03 \\
0.31^{\dagger} \\
-0.09 \\
0.26^{*} \\
0.32^{\dagger} \\
0.12 \\
0.23^{*} \\
0.24^{*} \\
-0.11 \\
0.14 \\
0.77^{\ddagger} \\
0.70^{\ddagger}\end{array}$ & $\begin{array}{c}-0.02 \\
0.44^{\ddagger} \\
-0.03 \\
0.32^{\dagger} \\
0.44^{\ddagger} \\
0.09 \\
0.35^{\ddagger} \\
0.18 \\
-0.28^{\dagger} \\
0.03 \\
0.80^{\ddagger} \\
0.74^{\ddagger} \\
0.80^{\ddagger}\end{array}$ & $\begin{array}{c}-0.13 \\
0.33^{\dagger} \\
-0.13 \\
0.37^{\ddagger} \\
0.40^{\ddagger} \\
0.16 \\
0.29^{\dagger} \\
0.23^{*} \\
-0.13 \\
0.11 \\
0.80^{\ddagger} \\
0.73^{\ddagger} \\
0.91^{\ddagger} \\
0.78^{\ddagger}\end{array}$ \\
\hline
\end{tabular}

${ }^{*} P<0.05 ;{ }^{\dagger} P<0.01 ;{ }^{\ddagger} P<0.001 . \mathrm{BMI}=$ body mass index; $\mathrm{Yrs}$ pm $=$ years postmenopause; $E 2$ = total oestradiol; Free E2 = free oestradiol index; Free $T=$ free testosterone index; DHEAS = dehydroepiandrosterone sulphate; SHBG = sex hormone binding globulin; $\mathrm{A}=$ androstenedione; $\mathrm{TBody}=$ total body mineral density; L2 - L4 = L2 - L4 bone mineral density; Neck = neck bone mineral density; Troc = trochanter bone mineral density; Ward = Ward's triangle bone mineral density. Bone mineral density is given in $\mathrm{g} / \mathrm{cm}^{2}$.

the mechanisms of bone loss among normal healthy women in the late postmenopause.

Studies examining the relationships between endogenous oestrogens and bone mass in postmenopausal women have given conflicting results. A number of studies have reported positive effects of endogenous oestradiol on spinal bone mineral density in pre- to early postmenopausal women, ${ }^{14,15}$ though this finding has not been universal. ${ }^{16}$ Both cross-sectional $^{17}$ and longitudinal studies, ${ }^{18}$ have demonstrated a positive relationship between oestrogen levels and radial bone mineral content. In contrast, a number of small studies among selected older women more than 5 years postmenopause failed to show any oestrogen effect on bone mass. ${ }^{22-24}$ We believe that our study is the first to show a positive relationship between endogenous free oestradiol levels and total body and hip bone mineral density among women in the late postmenopause. The biological plausibility of the relationships observed, and their consistency, supports the existence of a genuine relationship. Of interest, recent reports have indicated that hormone replacement therapy can retard bone loss or even increase bone mineral density in elderly women..$^{25,26}$

Although the relationships between the free oestradiol index and bone mass were reduced after adjusting for body mass index, an independent effect was still apparent at all sites except for the femoral neck. This partial confounding may be due to the aromatization of adrenal androgens to oestrone in adipose and skeletal tissue, which 
Table III Relationship of variables with bone mineral density by stepwise multiple regression analysis

\begin{tabular}{|c|c|c|c|c|}
\hline & $\begin{array}{c}\text { Beta } \\
\left(\mathrm{g} / \mathrm{cm}^{2}\right)\end{array}$ & $S E$ & $\boldsymbol{P}$ & $R^{2}$ \\
\hline \multicolumn{5}{|l|}{ 1. Total body BMD } \\
\hline BMI & 0.007 & 0.003 & 0.009 & 0.24 \\
\hline Yrs pm & -0.005 & 0.002 & 0.009 & 0.08 \\
\hline $\begin{array}{l}\text { Free E2 Index } \\
\text { 2. L2-L4 BMD }\end{array}$ & 0.055 & 0.026 & 0.04 & 0.04 \\
\hline $\begin{array}{l}\text { L2-L4 BMD } \\
\text { Free E2 Index }\end{array}$ & 0.154 & 0.041 & 0.0004 & 0.17 \\
\hline Yrs pm & -0.009 & 0.004 & 0.01 & 0.08 \\
\hline \multicolumn{5}{|l|}{ 3. Neck BMD } \\
\hline $\begin{array}{l}\text { Free E2 Index } \\
\text { 4. Troc BMD }\end{array}$ & 0.085 & 0.031 & 0.007 & 0.10 \\
\hline BMI & 0.011 & 0.003 & 0.0002 & 0.20 \\
\hline \multicolumn{5}{|l|}{ 5. Ward BMD } \\
\hline Free E2 Index & 0.135 & 0.038 & 0.0007 & 0.16 \\
\hline
\end{tabular}

BMD = bone mineral density $\left(\mathrm{g} / \mathrm{cm}^{2}\right) ; \mathrm{SE}=$ standard error; $\mathrm{BMI}=$ body mass index $\left(\mathrm{kg} / \mathrm{m}^{2}\right)$; Yrs $\mathrm{pm}=$ years postmenopausal; Free E2 Index = total oestradiol/sex hormone binding globulin; TBody BMD = total body BMD; Troc BMD = trochanter BMD; Ward BMD = Ward's triangle BMD.

subsequently is converted to oestradiol. ${ }^{27}$ The observed pan-skeletal effect of endogenous free oestradiol on bone mass is consistent with data showing that oestrogen replacement therapy prevents bone loss at all skeletal sites. ${ }^{28}$

We found a positive relationship between free rather than total oestradiol levels and bone mineral density. These findings are consistent with the widely held view that it is the unbound hormone which is biologically active. Additional supportive data were reported by Van Hemert et al. who found that sex hormone binding globulin was a powerful predictor of metacarpal bone loss among 746 postmenopausal women, an effect which was attributed to its modulating effect on free hormone levels. $^{29}$

The limitations of our study in addition to the modest sample size and cross-sectional design $\overline{0}$ should be acknowledged. Only one non-fasting $\frac{\overline{0}}{\square}$ serum sample was used to characterize each woman $\frac{\bar{\omega}}{\bar{D}}$ and measurement errors for the hormone assays were likely relatively large. We used a relatively crude estimate of free oestradiol levels and the oestradiol assay was not ideal for measuring the $\vec{\circ}$ low levels expected in postmenopausal women. However, these limitations would be expected to $\stackrel{\omega}{\circ}$ reduce the strength of the relationships observed rather than to produce spurious ones.

In summary, our results show a positive relationship between endogenous free oestradiol and total body, spine and hip bone mineral density in of postmenopausal women. These relationships were $\dot{\theta}$ modest, a one standard deviation increase in free $\infty$ oestradiol index being associated with a $6.4-7.6 \%$ 은 increase in bone mineral density. Nevertheless, these data suggest that even at low oestradiol $Z$ values and within the limited range, that endogenous free oestradiol appears to have a positive influence on bone mass even in the late postmenopause.

\section{Acknowledgements}

The authors thank Dr M.A. Laskey and the staff of the Bone Density Unit, Addenbrooke's Hospital for assistance with the bone mineral density measurements. This study was supported by grants from the Wellcome Trust, East Anglia Regional Health Authority, Wolfson Found- $\exists$ ation, and the Violet M. Richards Charity.

\section{References}

1. Cummings, S.R., Black, D.M., Nevitt, M.C. et al. Appendicular bone density and age predict hip fracture in women. JAMA 1990, 263: 665-668.

2. Hui, S.L., Slemenda, C.W. \& Johnson, C.C. Jr. Baseline measurement of bone mass predicts fracture in white women. Ann Intern Med 1989, 111: 355-361.

3. Elders, P.J.M., Netelenbos, J.C., Lips, P., van Ginkel, F.C. \& van der Stelt, P.F. Accelerated vertebral bone loss in relation to the menopause: a cross-sectional study on lumbar bone density in 286 women $46-55$ years of age. Bone Miner 1988, 5: 11- 19 .

4. Nilas, L. \& Christiansen, C. Rates of bone loss in normal women: evidence of accelerated trabecular loss after the menopause. Eur J Clin Invest 1988, 18: 529-534.

5. Munk-Jensen, M., Pors Nielsen, S., Obel, E.B. \& Bonne Eriksen, P. Reversal of postmenopausal vertebral bone loss by oestrogen and progestogen: a double blind placebo controlled trial. $\mathrm{Br}$ Med J 1988, 296: 1150-1152.

6. Lindsay, R. \& Tohme, J.F. Estrogen treatment of patients with established postmenopausal osteoporosis. Obstet Gynecol 1990, 76: 290-295.

7. Hutchinson, T.A., Polansky, S.M. \& Feinstein, A.R. Postmenopausal oestrogens protect against fractures of hip and distal radius: a case-control study. Lancet 1979, 2: 705-709.

8. Weiss, N.S., Ure, C.L., Ballard, J.H., Williams, A.R. \& Daling, J.R. Decreased risk of fractures of the hip and lower $\mathrm{S}$ forearm with postmenopausal use of estrogen. $N$ Engl J Med 1980, 303: 1195-1198.

9. Kiel, D.P., Felson, D.T., Anderson, J.J., Wilson, P.W.F. \& Moskowitz, M.A. Hip fracture and the use of estrogens in $N$ postmenopausal women: the Framingham Study. $N$ Engl $J$ Med 1987, 317: 1169-1174.

10. Marshall, D.H., Crilly, R.G. \& Nordin, B.E.C. Plasma N androstenedione and oestrone levels in normal and $\omega$ osteoporotic postmenopausal women. $\operatorname{Br}$ Med J 1977, ii: 1177-1179.

11. Riggs, B.L., Ryan, R.J., Wahner, H.W., Jiang, N.-S. \& Mattox, V.R. Serum concentrations of estrogen, testosterone and gonadotrophins in osteoporotic and nonosteoporotic postmenopausal women. J Clin Endocrinol Metab 1973, 36: $1097-1099$. 
12. Davidson, B.J., Riggs, B.L., Wahner, H.W. \& Judd, H.L. Endogenous cortisol and sex steroids in patients with osteoporotic spinal fractures. Obstet Gynecol 1983, 61: 275-278.

13. Davidson, B.J., Ross, R.K., Paganini-Hill, A., Hammond, G.D., Siiteri, P.K. \& Judd, H.L. Total and free estrogens and androgens in postmenopausal women with hip fractures. $J$ Clin Endocrinol Metab 1982, 54: 115-120.

14. Steinberg, K.K., Freni-Titulaer, L.W., DePuey, E.G. et al. Sex steroids and bone density in premenopausal and perimenopausal women. J Clin Endocrinol Metab 1989, 69: 533-539.

15. Johnson, C.C. Jr, Hui, S.L., Witt, R.M., Appledorn, R., Baker, R.S. \& Longcope, C. Early menopausal changes in bone mass and sex steroids. J Clin Endocrinol Metab 1985, 61: 905-911.

16. Spector, T.D., Thompson, P.W., Perry, L.A., McGarrigle, H.H. \& Edwards, A.C. The relationship between sex steroids and bone mineral content in women soon after the menopause. Clin Endocrinol 1991, 34: 37-41.

17. Cauley, J.A., Gutai, J.P., Sandler, R.B., LaPorte, R.E., Kuller, L.H. \& Sashin, D. The relationship of endogenous estrogen to bone density and bone area in normal postmenopausal women. Am J Epidemiol 1986, 124: 752-761.

18. Slemenda, C., Hui, S.L., Longcope, C. \& Johnson, C.C. Sex steroids and bone mass: a study of changes about the time of menopause. J Clin Invest 1987, 80: 1261-1269.

19. Nanjee, M.N. \& Wheeler, M.J. Plasma free testosterone - is an index sufficient? Ann Clin Biochem 1985, 22: 387-390.

20. Mazess, R.B., Barden, H.S., Bisek, J.P. \& Hanson, J. Dual-energy X-ray absorptiometry for total body and regional bone mineral and soft tissue composition. Am J Clin Nutr 1990, 51: 1106-1112.

21. Laskey, M.A., Flaxman, M.E., Barber, R.W. et al. Comparative performance in vitro and in vivo of Lunar DPX and Hologic QDR-1000 dual energy X-ray absorptiometers. Br J Radiol 1991, 64: 1023-1029.
22. Wild, R.A., Buchanan, J.R., Myers, C. \& Demers, L.M. Declining adrenal androgens: an association with bone loss in aging women. Proc Soc Exp Biol Med 1987, 186: 355-360.

23. Wild, R.A., Buchanan, J.R., Myers, C., Lloyd, T. \& Demers, L.M. Adrenal androgens, sex-hormone binding globulin and bone density in osteoporotic menopausal women: is there a relationship? Maturitas 1987, 9: 55-61.

24. Deutsch, S., Benjamin, F., Seltzer, V., Tafreshi, M., Kocheril, G. \& Frank, A. The correlation of serum estrogens and androgens with bone density in the late postmenopause. Int $J$ Gynaecol Obstet 1987, 25: 217-222.

25. Quigley, M.E.T., Martin, P.L., Burnier, A.M. \& Brooks, P. Estrogen therapy arrests bone loss in elderly women. $\mathrm{Am} \mathrm{J}$ Obstet Gynecol 1987, 156: 1516-1523.

26. Christiansen, C. \& Riis, B.J. 17ß-estradiol and continuous norethisterone: a unique treatment for established osteoporosis in elderly women. J Clin Endocrinol Metab 1991, 71: 836-841.

27. Longcope, C., Pratt, J.H., Schneider, S.H. \& Fineberg, S.E. Aromatization of androgens by muscle and adipose tissue in vivo. J Clin Endocrinol Metab 1978, 46: 146-152.

28. Gotfredsen, A., Nilas, L., Riis, B.J., Thomsen, K. \& Christiansen, C. Bone changes occurring spontaneously and caused by oestrogen in early postmenopausal women: a local or generalised phenomenon? Br Med J 1986, 292: 1098-1100.

29. Van Hemert, A.M., Birkenhager, J.C., De Jong, F.H., Vandenbroucke, J.P. \& Valkenburg, H.A. Sex hormone binding globulin in postmenopausal women: a predictor of osteoporosis superior to endogenous oestrogens. Clin Endocrinol 1989, 31: 499-509. 